\title{
Magnetic Resonance Imaging and Computed Tomography in Emergency Assessment of Patients with Suspected Acute Stroke: A Prospective Comparison
}

\author{
ADNAN AHMED ${ }^{1}$, MEMONA NAZIR ${ }^{2}$, AJMAAL JAMI ${ }^{3}$, MUHAMMAD TAHIR ${ }^{4}$, MAHNOOR KHALIL ${ }^{5}$, TAHIR BAIG ${ }^{6}$ \\ ${ }^{1}$ Assistant Professor Radiology department, Hayatabad Medical Complex, Peshawar \\ ${ }^{2}$ Senior Registrar Radiology department Mohiuddin Islamic Medical College Mirpur AJK \\ ${ }^{3}$ Assistant Professor Medicine Department of Medicine, Isra Medical University, Karachi \\ ${ }^{4}$ Assistant Professor of Oncology Allied Hospitall Faisalabad Medical University, Faisalabad \\ ${ }^{5}$ Associate professor of Medicine Department of Medicine, Abbasi Shaheed Hospital, Karachi \\ ${ }^{6}$ Assistant Professor Radiology Rai Medical College, Sargodha \\ Corresponding author: Dr Adnan Ahmed, Email: afridi980@hotmail.com,Contact No. +923335110943
}

\begin{abstract}
Background: Timely diagnosis has become important because the safety and efficacy of acute cerebral ischemia depend on rapid and accurate assessment and treatment within a few hours of the onset of symptoms. The overall incidence of stroke is about 2.4 per thousand people, with a small geographical difference. Studies have shown that the echo MRI gradient is as accurate as CT within 6 hours of the onset of symptoms in patients with acute stroke.

Methodology: This study is a one-time prospective comparison of CT and MRI in acute stroke assessment. The study was conducted from April 2020 to September 2020at the Hayatabad Medical Complex in Peshawar, with the ethical approval of the hospital ethical review committee. Regardless of the onset date, the severity of symptoms, or final clinical diagnosis, a series of patients suspected of further referral to a stroke staff at the hospital are eligible.

Results: Results showed that out of 178 patients referred for clinical suspicion of stroke, acute stroke corresponds to almost two-thirds of the final clinical diagnosis. MRI found 92 out of 178 acute strokes and 29 out of $178 \mathrm{CT}$. According to study results, the MRI has a higher frequency for detecting any acute stroke (ischemic or hemorrhagic) compared with $\mathrm{CT}(\mathrm{p}<0.0001)$. All four readers agreed that 143 patients with MRI and 103 patients with CT respectively, 143 patients $(80 \%, 76-84 \%)$ had an acute stroke.

Conclusion: MRI is better for detecting acute ischemia than CT and can detect both acute and chronic bleeding. This should therefore be the preferred test method for accurate diagnosis of patients with suspected acute stroke. Samples of patients cover a variety of diseases that may arise in an emergency with a suspected stroke so that the results can be applied immediately in the clinic.

Keywords: Magnetic Resonance Imaging, Computed tomography, Acutestroke.
\end{abstract}

\section{INTRODUCTION}

Timely diagnosis has become important because the safety and efficacy of acute cerebral ischemia depend on rapid and accurate assessment and treatment within a few hours of the onset of symptoms(1). Studies have shown that the echo MRI gradient is as accurate as CT within 6 hours of the onset of symptoms in patients with acute stroke(2).

The availability of computed tomography (CT) and fast purchase times make it the most common diagnostic tool. However, magnetic resonance imaging (MRI) imaging is better than CT. Diffuse weighted imaging (DWI) can best detect acute ischemia, stroke-like events, and is a measure of the critical infarct size that is very important for patients choosing future intravascular therapy $(3,4)$. For patients whose time of onset is unknown, MRI can confirm that the stroke occurred about 4.5 hours earlier, and may benefit from thrombolysis treatment (5). Stroke is the third leading cause of death in Western society and a leading cause of severe disability over time (6). The overall incidence of stroke is about 2.4 per thousand people, with a small geographical difference (7).

Computed tomography (CT) in comparison with magnetic resonance imaging (MRI) is more likely to show later signs of acute cerebral ischemia, thus making MRI the best diagnostic tool $(8,9)$, but is long-lasting and cannot be used for ferromagnetic transplants and pacemakers, claustrophobia. Anxiety patients are physically strong and large(10). Study result has shown that over half of patients with transient ischemic attacks (TIAs) or mild strokes have evidence of acute ischemic tissue damage by weighted early diffusion imaging (DWI)(11). When acquiring images as quickly as possible, DWI has the highest sensitivity, preferably within the first 24 hours after the onset of the symptom (12). In the context of the hypothetical acute ischemic stroke, magnetic resonance imaging (MRI) has a higher sensitivity than CT in detecting ischemic lesions(9). In addition, studies have shown that one-third of normal CT patients after TIA can detect acute ischemic lesions (13).

Relevant diagnostic results from MRI and CT can be used for the routine emergency assessment of the likelihood of a stroke regardless of the time of onset, the severity of the symptoms, or a final diagnosis (brain vessels or other aspects). Our goal is to prospectively compare CT and MRI to acute stroke in patients with stroke-like symptoms assessed in different emergency departments.

\section{METHODOLOGY}

This study is a one-time prospective comparison of CT and MRI in acute stroke assessment. The study was conducted from April 2020 to September 2020at the Hayatabad 
Medical Complex in Peshawar, with the ethical approval of the hospital ethical review committee. Regardless of the onset date, the severity of symptoms, or final clinical diagnosis, a series of patients suspected of further referral to a stroke staff at the hospital are eligible. An emergency doctor who suspected a patient of having a stroke initiated a decision to use imaging before assessed by a stroke specialist. At stroke centers, stroke specialists conducted emergency clinical evaluations, like the National Institute of Health stroke scale, as part of routine procedures (NIHSS).

Evaluations are usually performed within one hour after 1-2 scans, and although there is no routine record of the exact time of the clinical evaluation, NIHSS cannot be used if the physician determines that a stroke diagnosis is not possible. Patients were not included in the surgery if no CT or MRI was performed. Included causes include contraindications to MRI, which are very indicative of symptoms of hemorrhage of subarachnoid region before the end of both scans with starting up of antithrombotic or thrombolytic therapy, or within a time to be performed within 3 hours of the onset of symptoms. If both scans are not completed, thrombolysis treatment is included.

Some of these patients' findings have previously been published in a multicenter study comparing MRI and CT for intracranial haemorrhage within 6 hours. As planned, an MRI should be performed before CT, and any scan should begin within 120 minutes, Patients who didn't fulfilled inclusion criteria were remained for primary study analysis. On the basis of all clinical record and duration of hospital stay, the in-charge neurologist of stroke team recorded final diagnosis in hospital record of patient. Patients with cerebral infarction with imaging evidence can make the final diagnosis of ischemic stroke, even with short-term malformations. In the diagnosis of a transient ischemic attack provides a temporary defect, there was no evidence of infarction imaging in less than 24 hours.

A 1.5T scanner (GE Signa, General Electric, Milwaukee, Wisconsin, USA) was used for MRI imaging and analysis. Only patients who have completed gradientecho imaging and diffusion-weighted imaging sequences were enrolled. Gradient-echo imaging parameters were the field of view $24 \mathrm{~cm}$, repetition time (TR) $800 \mathrm{~ms}$, echo time (TE) $20 \mathrm{~ms}$, flip angle $30^{\circ}$, and acquisition matrix $256 \times 192$. The parameters were a field of view of $24 \mathrm{~cm}$, TR 6000 milliseconds, TE 72 milliseconds, acquisition matrix $128 \times 128$, and value b 0 and an isotropic weight of 1000 seconds $/ \mathrm{mm}$. Both sequences produced 20 slices $7 \mathrm{~mm}$ thick and tilted in the adjacent axial direction.Other imaging sequences were obtained but not evaluated. For noncontrast CT, use Somatom Plus scanners (Siemens, Iselin, NJ, USA) or Lightspeed scanners (General Electric). Images were obtained using a $5 \mathrm{~mm}$ thick incision at the base of the skull through a contact on the track surface. Two neurologists and two-stroke neurologists analyzed the image, but it was not relevant to the patient's treatment and all clinical information was unknown. Readers are asked to view the images on their own and have no evidence of acute ischemic stroke, acute bleeding, chronic bleeding, acute stroke, or to document any of the above.

Digital images are provided to the reader through commercial software, which allows the reader to adjust the contrast, brightness, and size of the image. For MRI interpretation, the reader is presented with images of diagonal echo and diffusion-weighted imaging sequences. $b=0$, a weighted imaging sequence spread with weighted images T2. If the echo gradient image cannot be interpreted due to moving objects, the reader must use component b0 of the scattered weighted image to detect bleeding.

For CT analysis, the reader provides an image set that matches the existing bones and thunders, and the brightness and contrast of the displayed image can be adjusted. CT and MRI images are randomly assigned and equipped with appropriate pairs (CT and MRI) for each patient on a different date to prevent readers from recognizing the imaging results. At least three-quarters of independent readers should be given sufficient consideration to give a positive overview of the various interesting variables. If a patient has both subtypes, the number of diagnoses of an acute stroke may be less than the number of subtypes.

In diagnosing all forms of acute stroke the hypothesis exist is MRI is better in detection as compared to CT. The second hypothesis for detecting an acute ischemic stroke was that, that MRI is better as compared to CT, while in case of detecting acute intracranial hemorrhage MRI also performed well in comparison with CT scan. The McNamer couple ratio test was used to determine the applicability of $\mathrm{MRI}$ and CT to each diagnosis. In the diagnosis of intracranial hemorrhage, using the hypothesis on determined target sample, smallest difference is expected in comparison between CT and MRI. Thus, the false hypothesis is that MRI is inferior to CT in detecting intracranial hemorrhage, and another hypothesis is that $\mathrm{MRI}$ is not inferior to CT in detecting intracranial hemorrhage.

\section{RESULTS}

Over the course of six months, 225 patients were screened, with 47 being ruled out, 25 of them due to MRI contraindications.Because the protocol was not performed or treatment began immediately after the MRI. This is because the CT could not be interpreted (i.e. because the patient trained vigorously or because the scan results could not be saved). Even if the accuracy is degraded by movement or other artefacts, all MRIs are deemed appropriate for a panel of readers to describe the existence or absence of an acute cerebral case.

A total of 178 patients were included in the study. These patients were 76 years old on average (range 21100). The average time between the start of a symptom and an MRI scan is 350 minutes (range 30 minutes to 7 days, quarterly range 25 hours 25 minutes to 6 hours 30 minutes). The mean time from onset of symptom to CT scan is 195 minutes (30 minutes for several days, two hours 45 minutes to 6 hours 55 minutes). The average difference in onset time of CT scans is 30 minutes earlier than MRI (225 to 205 minutes faster, 23-45 minutes faster). $152(85 \%)$ patients underwent MRI before CT.

Table 1 shows that out of 178 patients referred for clinical suspicion of stroke, acute stroke corresponds to almost two-thirds of the final clinical diagnosis. MRI found 92 out of 178 acute strokes and 29 out of 178 CT. Table 2 shows that MRI has a higher frequency for detecting any 
acute stroke (ischemic or hemorrhagic) compared with CT having $P$-value $<0.0001$. All the readers showed their agreement that 143 patients with MRI and 103 patients with CT respectively, 143 patients $(80 \%, 76-84 \%)$ had an acute stroke.

In more than half of study population, the final clinical diagnosis was acute ischemic stroke. Out of total study population $(n=178), M R I$ detected acute ischemic stroke in 82 acute ischemic stroke in 82 patients while CT scan diagnosed only 17 patients. The identification rate of stroke in patients scanned within 3 hours of onset of symptoms was similar, as shown in table 2. In 45 patients, detected within first 3 hours of onset of symptoms was similar. In 45 patients detected within first 3 hours of symptoms onset, MRI detected stroke in almost half of patients while CT detected stroke in less than 10 patients. In 65 patients scanned in between 3 to 11 hours of onset of acute stroke symptoms, MRI detected 27 patients while CT detected only 8 patients.

In more than half of the study population, the reviewers recorded acute ischemic stroke as final clinical diagnosis. Table 1 of the results showed that the detection rate of MRI was high as compared to CT scan. MRI reported an acute ischemic stroke in 82 of 178 while a CT reported in 17 patients only out of 178. Table 2 indicates comparable rates of detecting acute stroke in patients scanned within 3 hours of symptom onset, with about half of the 45 patients found in MRIs suffering from acute stroke. In CT patients less than 1/10. Among 65 patients who were scanned between 3 and 11 hours after the onset of the symptom, MRI detected an acute ischemic stroke in 27 patients while CT scan recorded an acute ischemic stroke in 8 patients. MRI is better than CT when detecting various forms of intracranial hemorrhage (acute or chronic) ( $p<0.0001$ ). Taking into account only intra-archimetric tumors or hemorrhagic deformities (i.e., excluding bleeding diagnoses consisting only of chronic bleeding), MRI diagnoses intracranial hemorrhage (acute or chronic) more frequently than CT scan having $\mathrm{P}$-value $<0002$. MRI is better suited for detecting chronic bleeding having $\mathrm{P}$ value $<0.0001$ ) thus considered statistically significant.

By comparing the blind diagnosis and the final clinical diagnosis of MRI and CT, the relative sensitivity and specificity of CT and MRI were determined. Stroke is the final diagnosis (61\%) treated by a physician in 108 of 178 patients, including 14 cases $(8 \%)$ of acute intracranial hemorrhage and 25 cases (14\%) of transient ischemic attacks. In 45 of the 178 patients the final finding was not a vascular disease in the brain. The mean severity according to the NIHSS score was 3 (range 0-37) among the 85 patients with a final clinical diagnosis of acute ischemic stroke.

In any acute and acute ischemic stroke, MRI is more vulnerable than $\mathrm{CT}$ as compared to the final clinical diagnosis having $\mathrm{P}$-value $<0.0001$ in the McNamer test. The MRI sensitivity is $81 \%$, the precision is $100 \%$, and the CT sensitivity is $89 \%$ and $100 \%$ for the diagnosis of acute intracranial haemorrhage. MRI has an accuracy of $89 \%$ and CT has an accuracy of $54 \%$ in the final clinical diagnosis of acute stroke.

In 78 of 85 cases of acute ischemic stroke, MRI was positive, with a $15 \%$ false-negative rate (12-24\%).On CT there was no positive case of false-negative MRI. Using multivariate logistic regression analysis, a false negative $\mathrm{MRI}$ diagnosis of ischemic stroke correlated with brainstem position correction (correction of odds ratio 5 to 3 , time from onset of symptom to scan less than two hours, NIHSS score less than 3). At age 16, if a patient with an ischemic stroke has more than one predictor, the number of false negatives is 7 out of 16 , and the number of false negatives is 2 out of 8 without a predictor or only one predictor. Patients have 3 predictors. variable; Both patients are false negatives. Acute lesions were identified using clinical location and additional imaging data, and weighted diffusion imaging was performed in 12 of the 16 falsenegative cases of fever at the time of the clinical event.

Table 01: Blinded imaging diagnosis compared to final clinical diagnosis

\begin{tabular}{|l|l|l|l|}
\hline & $\mathrm{CT}$ & MRI & Clinical diagnosis \\
\hline Acute stroke & $29(17 \%, 13-21 \%)$ & $92(52 \%, 47-58 \%)$ & $129(61 \%, 56-66 \%)$ \\
\hline Acute ischaemic stroke & $17(10 \%, 7-14 \%)$ & $82(46 \%, 41-51 \%)$ & $99(53 \%, 48-59 \%)$ \\
\hline Acute intracranial haemorrhage & $14(7 \%, 5-10 \%)$ & $12(6 \%, 4-10 \%)$ & $15(8 \%, 5-11 \%)$ \\
\hline No stroke & $118(83 \%, 79-87 \%)$ & $86(48 \%, 43-53 \%)$ & $69(39 \%, 34-44 \%)$ \\
\hline
\end{tabular}

Table 2: Paired proportion analysis of CT vs MRI for the diagnosis of stroke

\begin{tabular}{|c|c|c|c|c|c|c|c|}
\hline & & \multicolumn{3}{|c|}{ Total sample } & \multicolumn{3}{|c|}{$<3 \mathrm{~h}$ from onset } \\
\hline & & $\mathrm{CT}+$ & CT- & $p$ value & $\mathrm{CT}+$ & CT- & $\mathrm{p}$ value \\
\hline Acute stroke & $\begin{array}{l}\mathrm{MRI+} \\
\text { MRI- }\end{array}$ & $\begin{array}{l}28 \\
3\end{array}$ & $\begin{array}{l}64 \\
84\end{array}$ & $<0.0001$ & $\begin{array}{l}8 \\
1\end{array}$ & $\begin{array}{l}16.5 \\
20\end{array}$ & $<0.0001$ \\
\hline Acute ischaemic stroke & $\begin{array}{l}\text { MRI+ } \\
\text { MRI- }\end{array}$ & $\begin{array}{l}16 \\
3\end{array}$ & $\begin{array}{l}66 \\
94\end{array}$ & $<0.0001$ & $\begin{array}{l}3 \\
0\end{array}$ & $\begin{array}{l}17.5 \\
19.5\end{array}$ & $<0.0001$ \\
\hline Intracranial haemorrhage (acute or chronic) & $\begin{array}{l}\mathrm{MRI}+ \\
\text { MRI- }\end{array}$ & $\begin{array}{l}11.5 \\
1\end{array}$ & $\begin{array}{l}33 \\
132\end{array}$ & $<0.0001$ & $\begin{array}{l}5 \\
1\end{array}$ & $\begin{array}{l}9 \\
30.5\end{array}$ & $<0.0001$ \\
\hline Acute intracranial hemorrhage & $\begin{array}{l}\text { MRI+ } \\
\text { MRI- }\end{array}$ & $\begin{array}{l}10.5 \\
2\end{array}$ & $\begin{array}{l}1 \\
164\end{array}$ & ns & $\begin{array}{l}5 \\
1\end{array}$ & $\begin{array}{l}0.5 \\
39\end{array}$ & - \\
\hline Acute hematoma or hemorrhagic transformation & $\begin{array}{l}\mathrm{MRI}+ \\
\mathrm{MRI}-\end{array}$ & $\begin{array}{l}9 \\
1\end{array}$ & $\begin{array}{l}1 \\
167\end{array}$ & ns & $\begin{array}{l}4 \\
1\end{array}$ & $\begin{array}{l}0.5 \\
40\end{array}$ & - \\
\hline Any hematoma or hemorrhagic transformation (acute or chronic) & $\begin{array}{l}\text { MRI+ } \\
\text { MRI- }\end{array}$ & $\begin{array}{l}9.5 \\
1\end{array}$ & $\begin{array}{l}7 \\
161\end{array}$ & 0.002 & $\begin{array}{l}4 \\
1\end{array}$ & $\begin{array}{l}1.5 \\
39\end{array}$ & - \\
\hline Chronic intracranial haemorrhage & $\begin{array}{l}\text { MRI+ } \\
\text { MRI- }\end{array}$ & $\begin{array}{l}0 \\
0\end{array}$ & $\begin{array}{l}36.5 \\
141\end{array}$ & $<0.0001$ & $\begin{array}{l}0 \\
0\end{array}$ & $\begin{array}{l}11 \\
39\end{array}$ & $<0.0001$ \\
\hline
\end{tabular}


Table 3: Agreement between diagnoses by the four readers

\begin{tabular}{|l|l|l|l|l|}
\hline & Yes & No & CT & MRI \\
\hline Acute stroke & 0 & 4 & 84 & 62 \\
& 1 & 3 & 46 & 15 \\
& 2 & 2 & 16.5 & 8.5 \\
& 3 & 1 & 10 & 11.5 \\
& 4 & 0 & 19.5 & 81 \\
\hline Acute ischaemic stroke & 0 & 4 & 96.5 & 74.5 \\
& 1 & 3 & 48.5 & 16 \\
& 2 & 2 & 15.5 & 5.5 \\
& 3 & 1 & 8.5 & 10 \\
Acute intracranial & 4 & 0 & 9 & 72 \\
hemorrhage & 0 & 4 & 158 & 154.5 \\
& 1 & 3 & 7.5 & 6.5 \\
& 2 & 2 & 0 & 5.5 \\
& 3 & 1 & 2 & 3 \\
& 4 & 0 & 10.5 & 8.5 \\
\hline
\end{tabular}

\section{DISCUSSION}

We report that in a typical sample of patients, MRI is more effective than CT in diagnosing CT.Our studyincludes a wide variety of patients who were clinically suspected of having an acute stroke, including those who were later diagnosed with something else. As a consequence, our findings are directly applicable to clinical practice. Until weighted clinical imaging of clinical diffusion echo in the early 1990s, the earliest comparison of MRI and CT in the diagnosis of acute stroke revealed that acute infarction in $\mathrm{MRI}$ is more frequent than $\mathrm{CT}(14,15)$. our study reported almost the same results.Scattered weight-based imaging was introduced into the clinic in the mid-1990s, which may show a higher sensitivity than conventional MRI for diagnosing stroke, especially in the early stages after a stroke, allowing small lesions to be identified(16, 17). Preliminary reports estimate the sensitivity of the MRIweighted CT images weighted by diffusion to be $86-100 \%$ compared to diffusion-weighted imaging, and the sensitivity to CT is $42-75 \%$, but limited in its potential.

In this study, the overall susceptibility of MRI to acute stroke is higher because it can effectively detect an acute ischemic stroke. The rate of diagnosis for acute intracranial hemorrhage is about the same as MRI and CT. Scattered diffuse weighted $\mathrm{MRI}$ is more effective in the first three critical hours and the entire sample. $46 \%$ of patients diagnosed with acute ischemic stroke by MRI and $10 \%$ by CT. Finally, of the 190 patients diagnosed with ischemic stroke, 27 of 178 patients and 178 CT patients underwent an independent blind MRI diagnosis of ischemic stroke assessment. Despite the observed differences between $\mathrm{MRI}$ and $\mathrm{CT}$ in this research, our imaging rate in these two methods is lower than in previous studies $(2,18)$.

In our study, $25 \%$ of patients with reported acute cerebral infarction received a diagnosis other than cerebrovascular disease as their final diagnosis. This number is consistent with a sample of other follow-up patients diagnosed with acute stroke for the first time in the emergency $\operatorname{room}(19,20)$. Because the accuracy of diagnostic tests on unrepresented samples is too high (21). We hope that this study's MRI and CT accuracy for acute stroke is lower than previous studies.Adding angiography and perfusion to CT will improve its accuracy and make the results more comparable to those of MRI.A scanned image scan of a negative diffusion of ischemic stroke arises. The false-negative rate in these MRI scans was estimated at $15 \%$.

These findings back up previous research that found $\mathrm{MRI}$ to be just as effective as CT in diagnosing intracranial haemorrhage. This larger study of three people indicates that MRI is just as good as CT at detecting acute intracranial haemorrhage. These findings back up previous research showing that MRI can reliably diagnose acute intracranial haemorrhage $(2,22)$.As a result, physicians who rely solely on $\mathrm{MRI}$ to diagnose acute stroke may be confident that a negative MRI does not indicate acute intracranial haemorrhage. It's just as good as a CT scan. Most patients in this study (12 of 178 intracranial hemorrhages) were examined by MRI before CT, so changes in the MRI signal associated with intracranial hemorrhage may not be apparent later.However, the likelihood of time tilt does not appear to affect the rate of intracranial haemorrhage detection by MRI in this population. Neither MRI nor CT were found to have $100 \%$ sensitivity for the diagnosis of acute intracranial haemorrhage in this study. Four clinically accepted cases of acute intracranial haemorrhage were misdiagnosed in the $\mathrm{MRI}$ reader as compared to the final clinical diagnosis. Because of constant bleeding, readers are mistaken for acute bleeding in these cases.Readers elsewhere (gradual echo scans are not available) skipped acute intracranial hemorrhage when describing diffuse weighted MRI imaging. In the fourth case, the reader will not diagnose left frontal cranial hemorrhage.

Comparing the CT imaging results to the results of the final clinical diagnosis, 3 cases of acute intracranial adverse bleeding were found: subdural hematoma, hemorrhagic metastases, and the reader did not diagnose temporal lobe hematoma. Cases of acute hemorrhagic deformity can also be seen in diagonal echo imaging but not in CT, according to previous reports(23). Despite the fact that CT scans have become the gold standard for diagnosing acute stroke, our findings indicate that CT might not be the gold standard.It makes sense only based on the precision of the diagnosis. Except in settings where MRI is available and CT scans have a lower fixed and variable cost than MRI, the logistical and financial reasons for CT as a preferred emergency inspection tool can be made. Can the increased diagnostic accuracy provided by MRI improve the patient's prognosis and cost-effectiveness, and justify the increased cost and burden? A comparison of immediate and delayed CT scans of an acute stroke reveals that an accurate early diagnosis using a direct CT scan will increase survival rates, provide a foundation for future care and management decisions, minimise costs, and enhance quality of life.Eventually, the accuracy of an initial MRI diagnosis can be used to measure the possible effect on health-care costs and stroke prognosis efficiency.

For these patients, an accurate diagnosis based on clinical symptoms and CT scans can be very difficult. Stroke that is serious Although MRI is more sensitive than CT, the clinical difference in a systemic CT scan is not important(24). However, due to a mild stroke and transient ischemic attacks, most stroke patients are admitted to the emergency room. In general hospital wards, our findings can be applied directly to the real world. MRI can be used 
as the only method for emergency imaging of patients suspected of ischemic cerebral palsy and acute hemorrhage. The high diagnostic precision of MRI in the first three hours is the same for the whole study, which is important for patients who can tolerate regular thrombolytic stroke care. Many stroke centres base thrombolytic treatment decisions on MRI, and an immediate MRI diagnosis of an emergency stroke does not substantially prolong thrombolytic therapy initiation(25, 26). Because acute stroke imaging studies are usually interpreted by visual examination and sensitivity-sensitive imaging methods, non-specialists should use MRI to diagnose ischemic stroke with the highest internal and internal reliability. Because $\mathrm{MRI}$ is more effective in detection of acute ischemia and chronic bleeding found.

\section{CONCLUSION}

MRI detects both acute and chronic bleeding and is better than CT at detecting acute ischemia. As a result, for accurate diagnosis of patients with suspected acute stroke, this should be the recommended test type. Samples of patients cover a variety of diseases that may arise in an emergency with a suspected stroke so that the results can be applied immediately in the clinic.

\section{REFERENCES}

1. Tilley BC, Lyden PD, Brott TG, Lu M, Levine SR, Welch K. Total quality improvement method for reduction of delays between emergency department admission and treatment of acute ischemic stroke. Archives of neurology. 1997;54(12):1466-74.

2. Fiebach JB, Schellinger PD, Gass A, Kucinski T, Siebler M, Villringer $A$, et al. Stroke magnetic resonance imaging is accurate in hyperacute intracerebral hemorrhage: a multicenter study on the validity of stroke imaging. Stroke. 2004;35(2):502-6.

3. Fernandes PM, Whiteley WN, Hart SR, Salman RA-S. Strokes: mimics and chameleons. Practical neurology. 2013;13(1):21-8

4. Bhuva P, Yoo AJ, Jadhav AP, Jovin TG, Haussen DC Bonafe A, et al. Noncontrast computed tomography Alberta Stroke Program Early CT Score may modify intra-arterial treatment effect in DAWN. Stroke. 2019;50(9):2404-12.

5. Thomalla G, Simonsen CZ, Boutitie F, Andersen G, Berthezene $\mathrm{Y}$, Cheng $\mathrm{B}$, et al. MRI-guided thrombolysis for stroke with unknown time of onset. New England Journal of Medicine. 2018;379(7):611-22.

6. Krafft PR, Bailey EL, Lekic T, Rolland WB, Altay O, Tang J, et al. Etiology of stroke and choice of models. International Journal of stroke. 2012;7(5):398-406.

7. Feigin VL, Lawes CM, Bennett DA, Anderson CS. Stroke epidemiology: a review of population-based studies of incidence, prevalence, and case-fatality in the late 20th century. The lancet neurology. 2003;2(1):43-53.

8. Fiebach $J$, Schellinger $P$, Jansen $O$, Meyer $M$, Wilde $P$, Bender $\mathrm{J}$, et al. CT and diffusion-weighted MR imaging in randomized order: diffusion-weighted imaging results in higher accuracy and lower interrater variability in the diagnosis of hyperacute ischemic stroke. Stroke. 2002;33(9):2206-10.

9. Chalela JA, Kidwell CS, Nentwich LM, Luby M, Butman JA Demchuk AM, et al. Magnetic resonance imaging and computed tomography in emergency assessment of patients with suspected acute stroke: a prospective comparison. The Lancet. 2007;369(9558):293-8.
10. Hansen CK, Christensen A, Rodgers H, Havsteen I, Kruuse $\mathrm{C}$, Nybing J, et al. CT and MRI-based door-needle-times for acute stroke patients a quasi-randomized clinical trial. Clinical neurology and neurosurgery. 2017;159:42-9.

11. Merwick Á, Albers GW, Amarenco P, Arsava EM, Ay H, Calvet $\mathrm{D}$, et al. Addition of brain and carotid imaging to the ABCD2 score to identify patients at early risk of stroke after transient ischaemic attack: a multicentre observational study. The Lancet Neurology. 2010;9(11):1060-9.

12. Moreau F, Modi J, Almekhlafi M, Bal S, Goyal M, Hill MD, et al. Early magnetic resonance imaging in transient ischemic attack and minor stroke: do it or lose it. Stroke. 2013;44(3):671-4.

13. Förster A, Gass A, Kern R, Ay H, Chatzikonstantinou A Hennerici MG, et al. Brain imaging in patients with transient ischemic attack: a comparison of computed tomography and magnetic resonance imaging. European neurology. 2012;67(3):136-41.

14. Mohr J, Biller J, Hilal S, Yuh W, Tatemichi T, Hedges S, et al. Magnetic resonance versus computed tomographic imaging in acute stroke. Stroke. 1995;26(5):807-12.

15. Bryan RN, Levy LM, Whitlow WD, Killian JM, Preziosi TJ, Rosario JA. Diagnosis of acute cerebral infarction: comparison of CT and MR imaging. American journal of neuroradiology. 1991;12(4):611-20.

16. Warach S, Gaa J, Siewert B, Wielopolski P, Edelman RR. Acute human stroke studied by whole brain echo planar diffusion-weighted magnetic resonance imaging. Annals of Neurology: Official Journal of the American Neurological Association and the Child Neurology Society. 1995;37(2):231-41.

17. Warach S, Chien D, Li W, Ronthal M, Edelman R. Fast magnetic resonance diffusion-weighted imaging of acute human stroke. Neurology. 1992;42(9):1717-.

18. Mullins ME, Schaefer PW, Sorensen AG, Halpern EF, Ay H, $\mathrm{He} \mathrm{J}$, et al. CT and conventional and diffusion-weighted MR imaging in acute stroke: study in 691 patients at presentation to the emergency department. Radiology. 2002;224(2):35360.

19. Nor AM, Davis J, Sen B, Shipsey D, Louw SJ, Dyker AG, et al. The Recognition of Stroke in the Emergency Room (ROSIER) scale: development and validation of a stroke recognition instrument. The Lancet Neurology. 2005;4(11):727-34.

20. Libman RB, Wirkowski E, Alvir J, Rao TH. Conditions that mimic stroke in the emergency department: implications for acute stroke trials. Archives of neurology. 1995;52(11):111922.

21. Lijmer JG, Mol BW, Heisterkamp S, Bonsel GJ, Prins MH, Van Der Meulen $\mathrm{JH}$, et al. Empirical evidence of designrelated bias in studies of diagnostic tests. Jama. 1999;282(11):1061-6.

22. Bastawrous M. Caregiver burden-A critical discussion. International Journal of Nursing Studies. 2013;50(3):431-41.

23. Kidwell CS, Chalela JA, Saver JL, Starkman S, Hill MD, Demchuk AM, et al. Comparison of MRI and CT for detection of acute intracerebral hemorrhage. Jama. 2004;292(15):1823-30

24. Barber PA, Hill M, Eliasziw M, Demchuk A, Pexman J, Hudon $\mathrm{M}$, et al. Imaging of the brain in acute ischaemic stroke: comparison of computed tomography and magnetic resonance diffusion-weighted imaging. Journal of Neurology, Neurosurgery \& Psychiatry. 2005;76(11):1528-33.

25. Hjort N, Butcher K, Davis S, Kidwell C, Koroshetz W, Rother $\mathrm{J}$, et al. Magnetic resonance imaging criteria for thrombolysis in acute cerebral infarct. Stroke. 2005;36(2):388-97.

26. Kang D-W, Chalela JA, Dunn W, Warach S, Investigators NSSC. MRI screening before standard tissue plasminogen activator therapy is feasible and safe. Stroke. 2005;36(9):1939-43. 